\title{
IV. On the permanent state of heat in a thin uniform wire of any form, acted on by two sources of heat of equal intensity at its extremities
}

\section{G.W. Hearn B.A.}

To cite this article: G.W. Hearn B.A. (1846) IV. On the permanent state of heat in a thin uniform wire of any form, acted on by two sources of heat of equal intensity at its extremities, Philosophical Magazine Series 3, 29:191, 22-24, DOI: 10.1080/14786444608562591

To link to this article: http://dx.doi.org/10.1080/14786444608562591

曲 Published online: 30 Apr 2009.

Submit your article to this journal $[\pi$

Џll Article views: 2

Q View related articles $₫$ 


\section{[ 22$]$}

IV. On the Permanent State of Heat in a Thin Uniform Wire of any form, acted on by two sources of heat of equal intensity at its extremities. By G. W. Hearn, B.A. of Cambridge, and Professor of Mathematics, Royal Military College, Sandhurst*.

WTE suppose the wire homogeneous and of the same thickness throughout, $\theta^{2}$ the area of a transverse section, $\mathrm{K}$ the interior, and $h$ the exterior conductivity; $v$ the temperature at a point distant $s$ from one extremity measured along the wire.

The excess of the flux of heat between $s$ and $s+\delta s$, is

$$
k \varepsilon^{2} \frac{d^{2} v}{d s^{2}} \delta s
$$

and the quantity emitted by this portion is

$$
h c v \delta s \text {, }
$$

$c$ being the circumference of the transverse section,

$$
\begin{array}{ll}
\therefore \quad & \mathrm{K} \varepsilon^{2} \frac{d^{2} v}{d s^{2}}=h c v ; \\
& \frac{h c}{\overline{\mathbf{K} \varepsilon^{2}}}=k^{2}, \\
& \frac{d^{2} v}{d s^{2}}=k^{2} v, \\
& \therefore v=\mathbf{A} e^{-k s}+\mathbf{B} e^{k s} .
\end{array}
$$

Let the equal temperatures of the extremities be represented by 1 , and let $a$ be the length of the wire;

$$
\begin{aligned}
& \therefore \mathrm{A}+\mathrm{B}=1 \text {, } \\
& \mathrm{A} e^{-k a}+\mathrm{B} e^{k a}=1 \text {, } \\
& \mathbf{A}=\frac{e^{k a}-1}{e^{k a}-e^{-k a}}, \quad \mathbf{B}=\frac{1-e^{-k u}}{e^{k a}-e^{-k \omega}}, \\
& v=\mathrm{A}\left\{e^{-k s}+e^{-k(a-s)}\right\} \text {. . }
\end{aligned}
$$

Now suppose the temperature at the middle point to have been determined by observation $=t_{2}$.

Then

$$
\begin{gathered}
t_{2}=\mathbf{A}\left\{e^{-\frac{k a}{2}}+e^{-\frac{k n}{2}}\right\}=2 \mathrm{~A} e^{-\frac{k a}{2}}, \\
t_{2}=2 \frac{e^{k a}-1}{e^{k a}-e^{-k a}} \cdot e^{-\frac{k a}{2}}=\frac{2}{e^{\frac{k a}{2}}+e^{-\frac{k a}{2}}}
\end{gathered}
$$

from which

$$
e^{\frac{k a}{2}}=\frac{1+\sqrt{1-t_{2}^{2}}}{t_{2}} \text {. }
$$

* Communicated by the Author. 
Mr. G. W. Hearn on a permanent State of Heat. 23

Now the extreme temperatures being represented by 1 , it is evident that $t_{2}$ will be some proper fraction. Let therefore

$$
\begin{gathered}
t_{2}=\sin \alpha, \\
\therefore e^{\frac{k a}{2}}=\cot \frac{\alpha}{2}, \text { and } e^{-\frac{k a}{2}}=\tan \frac{\alpha}{2} .
\end{gathered}
$$

Hence

$$
\begin{gathered}
v=\frac{1}{2} t_{2} \cot \frac{\alpha}{2}\left\{\left(\tan \frac{\alpha}{2}\right)^{\frac{2 s}{a}}+\left(\tan \frac{\alpha}{2}\right)^{\frac{2(a-s)}{a}}\right\}, \\
v=\frac{1}{2} t_{2}\left\{\left(\tan \frac{\alpha}{2}\right)^{1-2 n}+\left(\cot \frac{\alpha}{2}\right)^{1-2 n}\right\}, \\
n=\frac{s}{a} .
\end{gathered}
$$

or

where

Now let $t_{3}$ be another observed temperature at the distance of $\frac{1}{3} a$ from either extremity,

$$
t_{3}=\frac{1}{2} t_{2}\left\{\left(\tan \frac{\alpha}{2}\right)^{\frac{1}{3}}+\left(\cot \frac{\alpha}{2}\right)^{\frac{1}{3}}\right\},
$$

whence

$$
\tan \frac{a}{2}=\frac{\left\{t_{3}-\sqrt{t_{3}^{2}-t_{2}^{2}}\right\}^{3}}{t_{3}^{3}}
$$

so that from those two observed temperatures we can easily calculate $\alpha$, and thence $v$.

To adapt the formulæ to logarithmic calculation, let

$$
\frac{t_{2}}{t_{3}}=\sin \eta
$$

then

$$
\tan \frac{\alpha}{2}=8 \sin ^{6} \frac{\eta}{2} \text {. }
$$

Moreover, make

$$
\left(\tan \frac{\alpha}{2}\right)^{1-2 n}=\tan \theta, \therefore\left(\cot \frac{\alpha}{2}\right)^{1-2 n}=\cot \theta,
$$

then

$$
v=t_{2} \operatorname{cosec} 2 \theta \text {. }
$$

Hence we have only to calculate

$$
\begin{aligned}
\log \sin \eta & =\log t_{2}-\log t_{3}, \\
\log \tan \theta & =(1-2 n)\left\{\log 8+6 \log \sin \frac{\eta}{2}\right\}, \\
\log v & =\log t_{2}+\log \operatorname{cosec} 2 \theta .
\end{aligned}
$$

It is also evident, since $e^{\frac{k a}{2}}=\cot \frac{\alpha}{2}$, we can obtain $k$, and thence $\frac{h}{\mathrm{~K}}$ the ratio of the exterior to the interior conductivity. 
24 Mr. G. W. Hearn on a permanent State of Heat.

The quantity $k$ will be usually very great, and $\therefore \alpha$ very small; and hence when $i<\frac{1}{2}$, we shall have very nearly

$$
v=\frac{1}{2} t_{2}\left(\cot \frac{\alpha}{2}\right)^{1-2 n}
$$

and when $n>\frac{1}{2}$,

$$
v=\frac{1}{2} t_{2}\left(\tan \frac{\alpha}{2}\right)^{1-2 n} \text {. }
$$

Let $v$ and $v^{\prime}$ be two temperatures corresponding to

$$
\begin{gathered}
n\left(<\frac{1}{2}\right) \text { and } n+\frac{1}{2} \\
\therefore v=\frac{1}{2} t_{2}\left(\cot \frac{\alpha}{2}\right)^{1-2 n}, v^{\prime}=\frac{1}{2} t_{2}\left(\tan \frac{\alpha}{2}\right)^{-2 n} ; \\
\therefore v v^{\prime}=\frac{1}{4} t_{2}^{2} \cot \frac{\alpha}{2} .
\end{gathered}
$$

Hence the product of the temperatures of points distant from each other by half the length of the wire (provided neither are very near the extremity) is nearly constant.

There is another curious relation which is not merely approximative, but accurately true, and which is easily verified by experiment. It is as follows:-

Having, as above determined, $\theta$, find $m$ from the formula

and then

$$
(\tan \theta)^{\frac{1-2 m}{1-2 n}}=\tan \left(\frac{\pi}{4}-\theta\right)
$$

and if $v$ and $v^{\prime}$ be the temperatures at $s$ and $s^{\prime}$,

$$
\begin{gathered}
v=t_{2} \operatorname{cosec} 2 \theta, \\
v^{\prime}=t_{2} \sec 2 \theta ; \\
\therefore \sin 2 \theta=\frac{t_{2}}{v^{\prime}}, \quad \cos 2 \theta=\frac{t_{2}}{v^{\prime}} ; \\
\therefore \frac{1}{v^{2}}+\frac{1}{v^{\prime 2}}=\frac{1}{t_{2}^{2}},
\end{gathered}
$$

or the sum of the reciprocals of the squares of the temperatures at such points is constant.

It is to be observed that Newton's law of cooling in vacuo is the basis of the above investigation. Also, that as the extreme temperatures of the wire are supposed equal, the two extremities may be joined and subjected to a single source of heat, in which case it will be a closed curve*.

* Vide Fourier, Théorie de la Chaleur, and Kelland on Heat. 